\title{
Educación ambiental en el nivel medio superior, desde la perspectiva de género, Tlaxcala, México'
}

\section{Environmental Education in Tlaxcala, Mexico, High Schools from a Gender Perspective}

\author{
Adelina Espejel-Rodríguez ${ }^{2}$ \\ Universidad Autónoma de Tlaxcala \\ Centro de investigaciones interdisciplinarias sobre desarrollo regional \\ Tlaxcala, México \\ adelinaer@hotmail.com \\ Aurelia Flores-Hernández ${ }^{3}$ \\ Universidad Autónoma de Tlaxcala \\ Centro de investigaciones interdisciplinarias sobre desarrollo regional \\ Tlaxcala, México \\ aure@hotmail.com \\ Isabel Castillo-Ramos 4 \\ Universidad Autónoma de Tlaxcala \\ Centro de investigaciones interdisciplinarias sobre desarrollo regional \\ Tlaxcala, México \\ icastillo@hotmail.com
}

Recibido 14 de enero de 2014 • Corregido 22 de mayo de 2014 • Aceptado 22 de agosto de 2014

\begin{abstract}
${ }^{1}$ Las autoras agradecen el financiamiento que el Cuerpo Académico (C.A-UATx-195) Estudios de Género, Educación y Juventud del CIISDER recibió a través del Programa Estratégico Cuerpos Académicos Calidad y Productividad para su Internacionalización de la Universidad Autónoma de Tlaxcala (CACyPI-UATx-2014). Por el cual fue posible la realización del presente trabajo.

${ }^{2}$ Es Doctora en Ciencias Económicas en la especialidad de Desarrollo Regional (área medio ambiente) por la Universidad de Camagüey, Cuba, becada por PROMEP y Maestra en Análisis Regional por la Universidad Autónoma de Tlaxcala (UATx). Pertenece al Sistema Nacional de Investigadores Nivel I y Perfil deseable del PROMEP/SEP. Trabaja la línea de educación, ambiente y género. Ha realizado diferentes proyectos de investigación apoyados por fondos sectoriales-mixtos, SEMARNAT- CONACYT, SIZA. Y ha publicado diversos artículos en revistas de cobertura nacional e internacional. Es autora de cinco libros y coautora de cuatro. Actualmente es profesora-investigadora en el Centro de Investigaciones Interdisciplinarias sobre el Desarrollo Regional (CIISDER) de la UATX.

${ }^{3}$ Es Doctora en Antropología por la Universidad Laval en Québec, Canadá. Miembro del Sistema Nacional de Investigadores Nivel I y Perfil deseable del PROMEP/SEP. Se ha especializado en estudios acerca de las mujeres rurales en el Centro Internacional MASHAV en Tel Avit, Israel. Es autora de varios libros entre ellos: A las mujeres por la "ley" no nos tocan tierras. Género, tierra, trabajo y migración (2010), Género y desarrollo. Problemas de la población I (2013) y co-autora de los libros: Ecología por competencias hacia una educación ambiental (2012) y Educación ambiental, fundamentos para la acción (2012). Actualmente es profesora-investigadora en el Centro de Investigaciones Interdisciplinarias sobre el Desarrollo Regional (CIISDER) de la Universidad Autónoma de Tlaxcala.

${ }^{4}$ Es Doctora en Ciencias Económicas, en la especialidad de Desarrollo Regional por la Universidad de Camagüey, Cuba, becada por PROMEP. Maestría en Análisis Regional por la Universidad Autónoma de Tlaxcala (UATx). Ha realizado diferentes proyectos de investigación apoyados por CONACYT, SIZA -UAT y el Instituto Interamericano de Cooperación para la agricultura (IICA). Es líder del Cuerpo Académico Análisis Regional y Estudios Metropolitanos. Se desempeña como profesora investigadora del CIISDER-UAT y sus publicaciones giran en torno a los temas de la participación de la mujer en proyectos productivos en contextos rurales y urbanos, temas de género, desarrollo, políticas públicas y ambiente.
\end{abstract}


doi: http://dx.doi.org/10.15359/ree.18-3.2

URL: http://www.una.ac.cr/educare

CORREO: educare@una.cr

Resumen. El trabajo tiene como objetivo describir las maneras en que el estudiantado -según su condición de género- innova acciones en favor del ambiente, para rescatar el papel de cambio para la promoción de la educación ambiental, asimismo las actividades que le motivan y le gusta realizar para conservar su escuela. La investigación se desarrolla en el marco de la metodología cualitativa, con una muestra de 80 estudiantes del bachillerato de ambos sexos, la información se obtiene a través de la observación directa, cuestionarios y las propias palabras del estudiantado. Esta se sistematiza y procesa para facilitar su análisis e interpretación, mediante el método de análisis descriptivo. Se encontró que hay diferencias y preferencias entre género para innovar y mitigar problemas, asimismo los motivos y el entusiasmo para llevar a cabo acciones ambientales. Una conclusión es que a las mujeres les gusta cuidar y conservar más su ambiente, en comparación con los hombres, ya que ellas muestran mayor interés en preservarlo.

Palabras claves. Educación ambiental, género, deterioro.

Abstract. This paper is aimed at describing how students, based on their gender, innovate actions in favor of the environment, in order to recover their role in the promotion of environmental education, as well as the activities that motivate them and that they like to maintain their school. The research is conducted within a qualitative methodology framework, with a sample of 80 male and female high school students. The information is collected using direct observation, questionnaires, and students opinions and is later systematized and processes for analysis and interpretation using a descriptive methodology. Results show differences and preferences between genders to innovate and mitigate problems, as well as differences in the reasoning and enthusiasm to perform environmental actions. One of the conclusions is that that women like to take care of the environment more than men since they show more interest in preserving it.

Keywords. Environmental education, gender, deterioration.

El modelo de desarrollo económico actual ha traído como consecuencia una serie de problemas ambientales, tales como pérdida de suelos y de recursos hídricos, contaminación de residuos sólidos peligrosos y no peligrosos, contaminación visual, cambio climático, deforestación y pérdida de la biodiversidad, los cuales han afectado gravemente los ecosistemas del planeta. De no afrontarse este deterioro a corto plazo, se pueden tener dificultades de escasez de los recursos naturales renovables y no renovables.

Es necesario contar con nuevas estrategias educativas desde la perspectiva de género para entender y mitigar, desde diversos puntos de vista, el deterioro ambiental de nuestro tiempo. La educación ambiental (EA) debe ser una herramienta elemental para que las personas adquieran conciencia de la importancia de preservar su entorno y sean capaces de realizar cambios en sus valores, conducta y estilos de vida, así como ampliar sus conocimientos para impulsarlas a la acción, mediante la prevención y mitigación de los problemas existentes y futuros. 
doi: http://dx.doi.org/10.15359/ree.18-3.2

URL: http://www.una.ac.cr/educare

CORREO: educare@una.cr

El presente trabajo tiene como objetivo describir las maneras en que el estudiantado -según su condición de género- innova acciones en favor del ambiente, para rescatar el papel de cambio que este tiene para la promoción de la educación ambiental; asimismo señalar las actividades ambientales que lo motiva y le gusta realizar para mitigar el deterioro ambiental de su escuela.

La investigación se desarrolló en el marco de la metodología cualitativa, a través de escritos, cuestionarios, observaciones directas y las propias palabras del estudiantado. Una de las conclusiones es que las mujeres tienen más cualidades para realizar acciones para combatir el desperdicio y contaminación de agua.

\section{Aspectos teóricos sobre el enfoque de género y ambiente}

La sociedad moderna atraviesa una seria crisis ambiental, con causas y efectos de diferentes dimensiones. Esto ha sido reconocido tanto en el ámbito nacional como internacional. La crisis ha deteriorado los ecosistemas del planeta, "sustento de los procesos económicos, sociales y culturales de las sociedades actuales, y que pone en riesgo la continuidad misma de la especie humana" (Velázquez, 1997, p. 55, en Arellano, 2003, p. 79).

La pérdida de los recursos naturales renovables y no renovables puede convertirse en irreversible si no se toman acciones concretas e inmediatas para mitigar los problemas ambientales como son la deforestación; la contaminación del aire, del agua, por basura; la pérdida de biodiversidad; el cambio climático; el adelgazamiento de la capa de ozono y su efecto invernadero.

Por tal motivo, en el aprendizaje del ambiente deben considerarse los objetivos de la educación ambiental (en adelante, EA), tales como:

Tratar la temática ambiental desde lo particular a lo general [con la] finalidad [sic]que los estudiantes se formen una idea de las condiciones ambientales [locales y regionales], además de que reflexionen sobre las dimensiones mundiales del problema ambiental para que los sujetos ... se involucren en los diferentes niveles de participación y responsabilidad [y] promover el conocimiento, la habilidad para solucionar problemas, la clasificación de valores, la investigación y la evaluación de situaciones, para aprender sobre la propia comunidad". (Martínez, 2010, p.101)

Ante esto, la protección, la conservación y el manejo del medio ambiente constituyen uno de los retos más importantes para la EA desde la perspectiva de género, concepción teórica y metodológica en la cual nos basamos en el presente estudio, ya que: 
doi: http://dx.doi.org/10.15359/ree.18-3.2

URL: http://www.una.ac.cr/educare

CORREO: educare@una.cr

El género es una categoría social, una atribución cultural de lo que se considera apropiado para cada sexo en una sociedad determinada, e incluye una serie de valores, roles y comportamientos. Las diferencias sociales y culturales entre el hombre y la mujer varían de una sociedad a otra $y$, al ser una construcción social, pueden modificarse. (Lamas, 1986, en Arellano 2003, p. 86)

La inclusión de la perspectiva de género... permite diferenciar las relaciones que hombres y mujeres establecen con los recursos naturales y los ecosistemas, pero, sobre todo, permite acceder a las formas de conocimiento y actitudes respecto del uso, acceso, control, beneficio, impacto y conservación de los recursos ..., en gran medida determinados por los valores sociales y estereotipos culturales. (Soares, Castorena y Ruiz, 2005, p. 68)

Aunque discutir de "género y medio ambiente como dos aspectos vinculados entre sí, ... no ha sido fácil hasta ahora, pese al auge [de] este tema a partir de los años ochenta" (Bifani-Richard, 2003, p. 8), ya se tienen "orientaciones conceptuales y metodológicas sobre cómo abordar la interrelación género, medio ambiente y desarrollo, [las que] genéricamente se denominan: a) ecofeminismo, b) mujeres y medio ambiente $y, c)$ género, medio ambiente y desarrollo sustentable" (Colombara, 2006, p. 167).

El ecofeminismo, corriente conocida por conceptualizar la relación de las mujeres con la naturaleza, "debido a sus experiencias biológicas o culturales, [estas]... son percibidas como 'naturalmente' cercanas a la naturaleza, lo cual, en el último de los casos, es resultado de las estructuras patriarcales en la sociedad, que subordinan la naturaleza a la cultura, y las mujeres a los hombres... (Diamond y Orenstein, 1990; Mies y Shiva, 1993; Shiva 1988)" (Pérez, 2003, pp. 283-284).

La corriente mujeres y medio ambiente "subraya el potencial del papel de las mujeres como administradoras de los recursos ambientales; su vulnerabilidad al cambio ambiental debido a su dependencia cercana con los recursos ambientales, y al hecho de que las iniciativas que se llevan a cabo sobre desarrollo y medio ambiente necesitan apoyar las contribuciones de las mujeres al medio ambiente (Collins, 1991; Dankelmann y Davidson, 1989; Paolisso, 1995)" (Pérez, 2003, p. 283).

La corriente género, medio ambiente y desarrollo sustentable considera la construcción de género como uno de los agentes intermediadores de las relaciones entre mujeres y varones con el medio ambiente y la sustentabilidad (Colombara, 2006). Nos suscribimos a esta última corriente porque nos permite explicar las diferencias de perspectivas existentes entre hombres y mujeres.

Las diferencias entre hombres y mujeres desde el enfoque de género son importantes, ya que estos a veces tienen intereses completamente distintos en lo que respecta al cambio ambiental (Joekes, Green y Leach, 2004). En este sentido, el enfoque permite visibilizar lo que normalmente no es visible (Tuñón, 2000). 


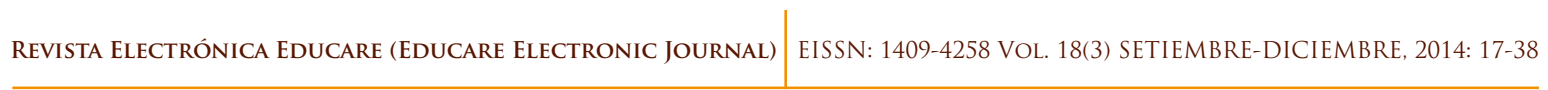

doi: http://dx.doi.org/10.15359/ree.18-3.2

URL: http://www.una.ac.cr/educare

CORREO: educare@una.cr

En este estudio interesa determinary diferenciar los intereses, motivaciones, innovaciones, visiones y estrategias que buscan para conservar y preservar su medio ambiente escolar los hombres y las mujeres. Para Lamas (1996, citado por Aranda y García, 2007, pp. 117-118):

La perspectiva de género busca reflexionar y comprender las características que definen a las mujeres y a los hombres de manera específica y diferenciada, con sus semejanzas, confrontaciones y puntos de encuentro... [así como] las diversas relaciones sociales [y culturales] que se dan entre ambos géneros. ... En una palabra, [interesa anotar] los recursos y la capacidad de acción con que cuentan mujeres y hombres para enfrentar las dificultades de la vida y la realización de propósitos [y proyectos].

Por otro lado, Bifani-Richard (2003, p. 9) agrega:

[Las] relaciones entre hombres y mujeres en un contexto social dado, nos ubican dentro de una situación real y no de una abstracción. Las mujeres no funcionan como una entidad separada, ajenas al tejido social, sino que lo hacen en interacción con los hombres. De ahí que sea importante determinar los roles que la sociedad les asigna a los unos y a los otros y las dificultades y potencialidades que ello significa".

Los roles de las mujeres y los hombres son distintos cuando se trata de cuidar, conservar y ejecutar acciones ambientales, ya que hay diferencias físicas, emocionales, de valores, sociales y culturales; sin embargo, ambos lo hacen en interacción de acuerdo con sus necesidades, motivaciones e intereses. Aunque a veces es difícil aceptar el control y la gestión de las actividades para el cuidado del ambiente, por las limitaciones y ventajas que tienen entre ellos para lograr sus objetivos.

La exploración del tema que nos ocupa implica no solamente entender las formas concretas del actuar de mujeres y hombres en las instituciones educativas con un programa ambiental, sino describir las maneras en que estudiantes -según su condición de génerorealizan acciones en favor del ambiente, para rescatar el papel de cambio que estos grupos tienen para la promoción de la educación ambiental; asimismo, para reconocer las actividades ambientales que les motivan y les gusta realizar para mitigar el deterioro ambiental y, de esta manera, producir un conjunto de conocimientos teóricos y prácticos desde la perspectiva de género para el cuidado y conservación del ambiente escolar.

A continuación se describen y analizan, desde la perspectiva de género, las actividades estudiantiles en los programas ambientales de la escuela, y los motivos y entusiasmo mostrado para mitigar problemas ambientales de su institución educativa.

\section{Orientación metodológica}

La investigación se desarrolla en el marco de la metodología cualitativa, definida por Taylor y Bogdan (1987) como "la investigación que produce datos descriptivos: las propias palabras de 
doi: http://dx.doi.org/10.15359/ree.18-3.2

URL: http://www.una.ac.cr/educare

CORREO: educare@una.cr

las personas, habladas o escritas, y la conducta observable" (p. 20). Esta empata con los fines de esta investigación, ya que a través de los escritos, observaciones directas y las propias palabras de estudiantes se puede recoger la información de acuerdo con los objetivos planteados.

Para la recolección de la información se utilizó la observación directa o participante, una técnica básica para realizar investigación cualitativa, consistente en "obtener impresiones del mundo circundante por medio de todas las facultades humanas relevantes" (Adler y Adler, 1998, en Álvarez-Gayou, 2012, p. 104). Esto suele requerir contacto directo con los sujetos. Taylor y Bogdan (1987) definen la observación participante como "la investigación que involucra la interacción social entre el investigador y los informantes ... [en] la cual se recogen datos de modo sistemático y no intrusivo" (p. 31).

Mediante las técnicas mencionadas, se obtiene la información de la siguiente manera:

a) Se observó la participación e interés del estudiantado en la ejecución de los programas ambientales (PA). La información obtenida se anotó de acuerdo con el objetivo de la investigación; sin embargo, para conseguir datos más fidedignos, se pidió al alumnado contestar un cuestionario, el cual se dividió en cuatro apartados: 1) aspectos generales, 2) actividades realizadas en sus PA, 3) organización y satisfacción de realizar acciones ambientales y 4) propuestas para obtener mejores resultados.

b) Como siguiente paso, se procedió al procesamiento, la sistematización y el análisis, utilizando el programa SPSS para facilitar su interpretación. Las observaciones y las respuestas dadas permitieron realizar la descripción y la reflexión sobre el tema. El método descriptivo fue el que se utilizó para el análisis de resultados.

Se seleccionó el plantel CBTis 212 de Tetla de la Solidaridad, localizado en el estado de Tlaxcala, ubicado geográficamente en la parte centro-oriental de la República Mexicana, para aplicar programas ambientales (PA) y así describir las maneras en que estudiantes -según su condición de género- innovan acciones en favor del ambiente, para rescatar el papel de cambio que ellos tienen para la promoción de la educación ambiental. Esta institución fue elegida por encontrarse ubicada en una ciudad con mayor número de problemas ambientales (Espejel, 2009).

Una vez seleccionada el área de estudio, se determinó aplicarlos en tres grupos del CBTis 212, al estudiantado que cursa la materia de ecología. Los grupos son seleccionados por el directivo del plantel, asignando a los grupos del $3^{\circ} \mathrm{A}$ y $3^{\circ} \mathrm{B}$ de la especialidad de mecatrónica e informática, turno matutino $(\mathrm{M})$ y vespertino $(\mathrm{V})$.

Un total de 80 estudiantes de ambos sexos diseñaron sus PA en el CBTis 212. Posteriormente, se realizó el procesamiento y análisis de la información. Los datos derivados del trabajo de campo se sistematizaron y procesaron para facilitar su análisis e interpretación. El método de análisis descriptivo se utilizó para indicar los resultados pertinentes del proyecto. 


\section{Resultados}

\section{Aspectos generales de los equipos conformados}

Para conocer y determinar cómo estudiantes de ambos sexos realizan acciones en favor del ambiente, se conformaron un total de 12 equipos del cuarto semestre de las especialidades de informática y mecatrónica del turno matutino y vespertino, para que diseñaran programas ambientales $^{5}$ (PA). Con la finalidad de conocer, desde la perspectiva de género las acciones y objetivos que se proponen en su PA para mitigar el deterioro ambiental de su escuela.

El total de estudiantes que realizaron PA fueron 80, de los cuales 45 fueron del sexo masculino y 35 del sexo femenino. Cada equipo se identificó con un nombre inspirado en cuestiones ecológicas (ver tabla 1).

Tabla 1

Aspectos generales de los equipos

\begin{tabular}{ccllccc}
\hline Grupo & Turno & Especialidad & Nombre del equipo & Total & Sexo masculino & Sexo femenino \\
\hline 4 B & V & Informática & Los rabanitos & 8 & 1 & 7 \\
4 A & V & Informática & Enanitos verdes & 8 & 2 & 6 \\
4 B & V & Informática & Ecologistas por el mundo & 10 & 5 & 5 \\
4 A & M & Informática & "LemonTree" & 9 & 4 & 5 \\
4 A & M & Informática & Los ecológicos & 9 & 6 & 3 \\
4 A & M & Informática & “Protectores de Gaia & 10 & 7 & 3 \\
4 A & M & Mecatrónica & Eco-clean & 5 & 5 & 0 \\
4 A & M & Mecatrónica & Sixpinos & & 7 & 0 \\
4 A & M & Mecatrónica & Green street & 7 & 7 & 1 \\
4 A & M & Mecatrónica & Huellitas verdes & 3 & 4 & 3 \\
4 A & M & Mecatrónica & Linternas verdes & 2 & 2 & 0 \\
4 A & M & Mecatrónica & Ecólogos & 4 & 2 & 2 \\
\hline Total & & & & 80 & 45 & 35 \\
\hline
\end{tabular}

Nota: Elaboración propia.

\footnotetext{
${ }^{5}$ Los programas ambientales "están conformados por un conjunto de acciones concretas y viables para aminorar el deterioro ambiental [de su escuela y comunidad], así como para que los estudiantes desarrollen conocimientos, valores, habilidades y competencias para conservar su ambiente" (Espejel y Flores 2012, p. 1175).
} 
doi: http://dx.doi.org/10.15359/ree.18-3.2

URL: http://www.una.ac.cr/educare

CORREO: educare@una.cr

El estudiantado conformó sus propios equipos, de los cuales ocho fueron mixtos, tres solo hombres y un solo equipo de mujeres. Como se puede ver, la mayor parte de los equipos fueron conformados por hombres y mujeres. Bifani-Richard (2003) menciona que "las mujeres no funcionan como una entidad separada, ajenas al tejido social, sino que lo hacen en interacción con los hombres. De ahí que sea importante determinar los roles que la sociedad les asigna a [las unas] y a los otros y las dificultades y potencialidades que ello significa" (p. 9).

Estudiantes de cada equipo elaboraron sus PA con actividades viables y congruentes con la realidad de la problemática ambiental de la escuela. Las acciones programadas fueron aplicadas en diversas áreas de su plantel educativo. El alumnado, en primer lugar, forma equipos y cada uno se identifica con un nombre relacionado con la naturaleza; posteriormente, planean su PA tratando de mitigar problemas ambientales detectados en la escuela, por observación directa y con la búsqueda de información bibliográfica. Luego, consensan las actividades por realizar en su programa, asimismo las fechas en que pueden llevarlas a cabo.

A continuación se plantean y analizan los PA que elaboró y aplicó el estudiantado en el CBTis 212. Se describen las actividades planeadas y realizadas por los equipos, el grado de preocupación del deterioro ambiental de su escuela y sus iniciativas para solucionarlo, desde la perspectiva de género.

\section{Diseño y aplicación de programas ambientales escolares desde la perspectiva de género}

Los alumnos y alumnas realizaron sus PA con diversas acciones para combatir los problemas ambientales de su escuela. Ya que "la EA no debe ser abstracta sino estar ligada a la realidad del entorno local y debe atender la formación de comportamientos responsables, para la creación de valores y actitudes positivas al ambiente (González, 2007, p. 40). Asimismo, "los programas de educación ambiental deben ser cuidadosamente dirigidos hacia las necesidades específicas locales" (Martínez, 2010, p. 104). El estudiantado concibe su PA como se menciona en el siguiente testimonio:

El programa ambiental consiste en un conjunto de acciones que realizamos con la idea de mejorar y contribuir con el medio ambiente, además de tener en cuenta la forma de pensar de las personas y la falta de valores respecto a lo que es el cuidado del ambiente (equipo los ecológicos).

Con base en esta concepción, el estudiantado diseñó sus programas ambientales escolares, con una serie de actividades planeadas para un determinado tiempo. 


\section{a) Descripción de las actividades ambientales plasmadas en los PA}

Cada equipo elaboró su PA escolar, con sus objetivos específicos relacionados, esencialmente, con informar y aprender sobre el cuidado del medio ambiente, mitigar problemas ambientales y hacer conciencia. Cada uno de estos propósitos los plasma con diferentes acciones ambientales, con la participación de hombres y mujeres.

Todos los equipos Ilevaron a cabo su PA en el CBTis 212, cada uno realizó diferentes actividades y se pudo observar la forma de innovar y hacer acciones según género. Por ejemplo El equipo "Los rabanitos" ejecutó las actividades planeadas en su PA durante el periodo mencionado (ver tabla 2). Cada actividad que realizaban tenían que demostrar con fotos el antes y el después, y hacer una bitácora, donde relataban todo lo sucedido, en el momento que realizaban sus acciones ambientales.

Tabla 2

Programa ambiental escolar del alumnado del CBTis 212

\begin{tabular}{|c|c|c|}
\hline Nombre del equipo & \multicolumn{2}{|l|}{ Los rabanitos } \\
\hline Programa ambiental escolar: & \multicolumn{2}{|l|}{ CBTis 212} \\
\hline Objetivo: & \multicolumn{2}{|c|}{$\begin{array}{l}\text { Enseñar al alumnado y personal docente a tener conciencia ambiental, as } \\
\text { como a fomentar una educación ambiental para lograr que el futuro del } \\
\text { planeta sea mejor y que el CBTis } 212 \text { sea una institución ecológica }\end{array}$} \\
\hline $\begin{array}{l}\text { Problemas ambientales de la } \\
\text { escuela: }\end{array}$ & Acciones ambientales por realizar, viables: & $\begin{array}{l}\text { Fecha de realización } \\
\qquad(2012)\end{array}$ \\
\hline $\begin{array}{l}\text { En los baños de mujeres } \\
\text { desperdician mucho el agua }\end{array}$ & $\begin{array}{l}\text { - Hacer carteles con un mensaje positivo respecto } \\
\text { al agua }\end{array}$ & 7 marzo \\
\hline Aula de clase sucia & - Hacer limpieza completa del salón de clases & 14 de marzo \\
\hline Deforestación & - Plantar árboles y plantas en el área de campo & 4 de abril \\
\hline \multirow[t]{2}{*}{$\begin{array}{l}\text { La cancha de futbol tiene } \\
\text { mucha basura }\end{array}$} & $\begin{array}{l}\text { - Poner un cartel para que no tiren basura en la } \\
\text { cancha }\end{array}$ & 18 de abril \\
\hline & - Recoger la basura de la cancha & \\
\hline Contaminación del agua & $\begin{array}{l}\text { Pedirles a los alumnos que ahorren el agua y no } \\
\text { tiren desechos por las coladeros }\end{array}$ & 2 de mayo \\
\hline \multirow[t]{3}{*}{ Contaminación de áreas verdes } & - Hacer faena & 30 de mayo \\
\hline & - Hacer un cartel de no tirar basura & \\
\hline & - Regar las plantas de las áreas verdes & \\
\hline
\end{tabular}

Nota: Elaboración propia. 
doi: http://dx.doi.org/10.15359/ree.18-3.2

URL: http://www.una.ac.cr/educare

CORREO: educare@una.cr

A continuación se muestran las descripciones de las actividades que realizaron integrantes del equipo Los rabanitos, de acuerdo a su PA, conformado por 1 hombre y 7 mujeres. Se puede ver que el trabajo se divide entre todos sin tomar en cuenta el sexo.

El 14 de marzo de 2012, nos pusimos de acuerdo el equipo "LOS RABANITOS", para tener limpio el salón, pues está demasiado sucio, fue una labor de mucho tiempo debido a que había mucha basura tirada por donde quiera y pues a nadie le gusta trabajar en un lugar sucio, así que nos dispusimos a cumplir nuestra actividad de tener un salón limpio pues sabíamos que teníamos que dejarlo impecable, recogimos la basura, barrimos el piso y limpiamos las ventanas. Al inicio de la actividad fue desesperante ya que había mucha basura y las ventanas estaban demasiado sucias. Todo el equipo "LOS RABANITOS"pusimos de nuestra parte para facilitar la limpieza del salón pidiéndole al policía de la caseta de la escuela que nos prestara unas cubetas, trapeador, trapo entre otras herramientas de limpieza.

Asíque todos nosotros nos repartimos el trabajo unos limpiando ventanas y otros barriendo el aula y, el trabajo fue más simple de realizar gracias a la cooperación de nuestro equipo "LOS RABANITOS". Nos llevamos una gran satisfacción al ver cómo había tenido un gran cambio nuestra aula de clase verla muy limpia y ver que así puede durar mucho tiempo el aula. Una vez terminada nuestra limpieza del salón llevamos las herramientas de limpieza a la caseta del policía dándole las gracias por habérnoslas prestado cumpliendo así con nuestra solución del problema de nuestra aula.

Este equipo, integrado por un hombre y varias mujeres, divide el trabajo entre todos sin importar el sexo, aunque el hombre, a veces, hacia las actividades de mayor fuerza. En la mayor parte de los equipos, la líder era una mujer, considerada por los integrantes como las más cooperativa y responsable; ella decía lo que tenía que hacer cada integrante del equipo. Los autores Cáceres y López (2009) mencionan que en el "liderazgo femenino comparado con el masculino (o la variable sexo) se ha encontrado que el segundo se percibe como más competitivo, controlador, analítico, jerárquico y no tiene en cuenta las emociones, en tanto que el femenino, por el contrario, tiende a ser más colaborativo, emotivo y cooperativo" (p. 586).

\section{b) Problemas ambientales que mitigaron los estudiantes desde la perspectiva de género}

Los problemas ambientales escolares que combaten los jóvenes y las jóvenes del nivel medio superior son: la contaminación de residuos sólidos (basura), áreas verdes maltratadas, desperdicio y contaminación del agua, falta de valores ambientales, contaminación visual y deforestación. Para cada problema ambiental establecen y ejecutan acciones ambientales, el mayor número de estas las realizan para combatir problemas de contaminación de residuos sólidos y áreas verdes. 
doi: http://dx.doi.org/10.15359/ree.18-3.2

URL: http://www.una.ac.cr/educare

CORREO: educare@una.cr

Las mujeres en comparación con hombres plasman más acciones para combatir el desperdicio y contaminación de agua y contaminación visual (ver tabla 3). Esto tiene relación con lo que dice Soares (2007), acerca de que las mujeres cuidan más el agua porque son las que "conocen, más que nadie, las demandas reales de las unidades domésticas en lo tocante al agua, por la sencilla razón de que son las responsables de manejar el agua dentro del hogar, ya sea en el lavado de ropa, preparación de alimentos o aseo de la vivienda, entre otras actividades" (p. 42).

Tabla 3

Problemas ambientales que combate el estudiantado en la escuela, según género

\begin{tabular}{lclc}
\hline \multicolumn{1}{c}{ Hombres } & \multicolumn{2}{c}{ Mujeres } \\
\hline Problemas ambiental de la escuela & $\begin{array}{c}\text { \% de acciones } \\
\text { ambientales }\end{array}$ & $\begin{array}{l}\text { Problema ambientales de la } \\
\text { escuela }\end{array}$ & $\begin{array}{c}\text { \% de acciones } \\
\text { ambientales }\end{array}$ \\
\hline $\begin{array}{l}\text { Contaminación de residuos } \\
\text { sólidos }\end{array}$ & 23 & $\begin{array}{l}\text { Contaminación de residuos } \\
\text { sólidos }\end{array}$ & 20 \\
Áreas verdes maltratadas & 21 & Áreas verdes maltratadas & 20 \\
Deforestación & 18 & Deforestación & 16 \\
$\begin{array}{l}\text { Desperdicio y contaminación de } \\
\text { agua }\end{array}$ & 14 & $\begin{array}{l}\text { Desperdicio y contaminación } \\
\text { de agua }\end{array}$ & 18 \\
Contaminación visual & 9 & Contaminación visual & 12 \\
Falta de valores ambientales & 14 & Falta de valores ambientales & 14 \\
\hline
\end{tabular}

Nota: Elaboración propia.

El estudiantado realiza acciones diversificadas para mitigar el problema de áreas verdes maltratadas y menos acciones para mitigar los problemas de deforestación y contaminación visual, cabe mencionar que los hombres diversifican más que las mujeres, esto debido que en gran parte de las actividades se necesita la fuerza física del hombre. Es importante señalar que la institución (CBTis 212) tiene grandes extensiones de áreas verdes (principalmente jardineras y setos de árboles), lo que hace atractivo para que los grupos de jóvenes realicen sus acciones en estos espacios. La escuela no cuenta con área boscosa, ya que el clima y el suelo son áridos.

Cada equipo se organiza para llevar a cabo sus acciones ambientales, en el tabla 4 se puede observar que tanto hombres como mujeres realizaron las mismas actividades, solo difieren en algunas. 
doi: http://dx.doi.org/10.15359/ree.18-3.2

URL: http://www.una.ac.cr/educare

CORREO: educare@una.cr

Tabla 4

Realización de actividades ambientales en la escuela según equipo y sexo

\begin{tabular}{|c|c|c|c|c|}
\hline $\begin{array}{l}\text { Nombre } \\
\text { del equipo }\end{array}$ & Hombres & Mujeres & Ambos & Y el por qué \\
\hline Los Rabanitos & $\begin{array}{l}\text { - Cargar cubetas con } \\
\text { agua } \\
\text { - Cargar las bolsas de } \\
\text { basura }\end{array}$ & $\begin{array}{l}\text { - Platicar con los niños } \\
\text { - Recoger basura } \\
\text { - Quitar la yerba de los } \\
\text { arbolitos }\end{array}$ & & $\begin{array}{l}\text { - El hombre hizo los trabajos más } \\
\text { pesados, porque tiene más fuerza }\end{array}$ \\
\hline $\begin{array}{l}\text { Ecologistas por } \\
\text { el mundo }\end{array}$ & $\begin{array}{l}\text { - Cavar los hoyos para las } \\
\text { plantas } \\
\text { - Plantar las flores } \\
\text { - Sacar toda la tierra }\end{array}$ & $\begin{array}{l}\text { - Regar las plantas } \\
\text { - Podar los arbustos } \\
\text { - Recoger basura } \\
\text { - Cargar cubetas de agua }\end{array}$ & $\begin{array}{l}\text { - Levantar basura } \\
\text { - Traer árboles, rosas, } \\
\text { y plantas }\end{array}$ & $\begin{array}{l}\text { - Algunas actividades son para } \\
\text { mujeres y otras para hombres. } \\
\text { - Nos repartimos el trabajo y todos } \\
\text { colaboramos }\end{array}$ \\
\hline Enanitos verdes & $\begin{array}{l}\text { - Plantar árboles } \\
\text { - Hacer los hoyos }\end{array}$ & - Recoger basura & $\begin{array}{l}\text { - Dar pláticas } \\
\text { - Recolectar botellas }\end{array}$ & $\begin{array}{l}\text { - Ambos hicimos lo mismo, aunque } \\
\text { los hombres plantaron e hicieron } \\
\text { los hoyos }\end{array}$ \\
\hline Lemon tree & & & $\begin{array}{l}\text { - Cortar el pasto } \\
\text { - Podar árboles } \\
\text { - Recolectar basura } \\
\text { - Hacer cepas } \\
\text { - Echar cal a los } \\
\text { árboles, } \\
\text { - Regar plantas } \\
\text { - Plantar flores }\end{array}$ & $\begin{array}{l}\text { - Nosotros nos consideramos como } \\
\text { iguales, todos hacemos de todo, } \\
\text { no importa si somos mujeres u } \\
\text { hombres. } \\
\text { - Todos hemos sido equitativos en } \\
\text { todas las actividades para evitar } \\
\text { problemas }\end{array}$ \\
\hline Los ecológicos & $\begin{array}{l}\text { - Arrancar la maleza } \\
\text { - Sacudir arbustos que } \\
\text { tenían telarañas y } \\
\text { basura. } \\
\text { - Hacer orificios para } \\
\text { plantar los árboles y } \\
\text { plantas. }\end{array}$ & $\begin{array}{l}\text { - Limpiar áreas verdes de } \\
\text { la parte de la fuente } \\
\text { - Recoger y limpiar la } \\
\text { maleza y el pasto } \\
\text { - Barrer y lavar la fuente }\end{array}$ & $\begin{array}{l}\text { - Limpiar el salón } \\
\text { - Hacer carteles } \\
\text { - Cortar arbustos } \\
\text { - Plantar árboles } \\
\text { - Barrer el salón } \\
\text { - Recolectar la basura }\end{array}$ & $\begin{array}{l}\text { - Porque ellos tiene más fuerza que } \\
\text { nosotros y decidimos que ellos } \\
\text { plantaran las plantas. } \\
\text { - A las mujeres les gusta más barrer y } \\
\text { estar en la sombra } \\
\text { - Hay cosas que pueden hacer los } \\
\text { hombres por su fuerza o porque no } \\
\text { les tienen miedo a los animales }\end{array}$ \\
\hline $\begin{array}{l}\text { Protectores de } \\
\text { Gaia }\end{array}$ & $\begin{array}{l}\text { - Ordenar las piedras } \\
\text { - Limpiar el área } \\
\text { - Acomodar piedras } \\
\text { - Recoger varas y troncos }\end{array}$ & $\begin{array}{l}\text { - Recoger basura } \\
\text { - Recoger la basura en } \\
\text { bolsas y tomar fotos } \\
\text { para la evidencia }\end{array}$ & $\begin{array}{l}\text { - Deshierbar y } \\
\text { recoger basura. } \\
\text {-Hacer cosas pesadas } \\
\text { - Reforestar } \\
\text { - Hacer hoyos }\end{array}$ & $\begin{array}{l}\text { - Los chavos tienen más fuerza física. } \\
\text { - Se vería muy raro que las mujeres } \\
\text { realicen trabajo pesado } \\
\text { - Para protegerlas y evitar accidentes }\end{array}$ \\
\hline Green street & $\begin{array}{l}\text { - Separar y reciclar la } \\
\text { basura recolectada }\end{array}$ & $\begin{array}{l}\text { - Atraer la atención de } \\
\text { las personas para } \\
\text { enseñarles cómo se } \\
\text { debe aprovechar y } \\
\text { reutilizar la basura }\end{array}$ & & $\begin{array}{l}\text { - Porque la integrante no podía } \\
\text { realizar esto ya que daría un mal } \\
\text { aspecto al equipo. M: para cuidar el } \\
\text { medio ambiente }\end{array}$ \\
\hline Eco clean & $\begin{array}{l}\text { - Hacer una campaña de } \\
\text { limpieza } \\
\text { - Mantener jardineras } \\
\text { - Pintar árboles con cal } \\
\text { - Hacer carteles } \\
\text { - Colocar botes } \\
\text { - Elaborar encuestas }\end{array}$ & & & $\begin{array}{l}\text { - Todos somos hombres y todos } \\
\text { participamos }\end{array}$ \\
\hline Eco CBETIS & $\begin{array}{l}\text { - Juntar basura } \\
\text { - Colocar botes de } \\
\text { basura } \\
\text { - Colocar carteles } \\
\text { - Regar árboles } \\
\text { - Entregar trípticos } \\
\text { - Plantar árboles } \\
\text { - Encalar los árboles } \\
\text { - Podar los jardines }\end{array}$ & & & $\begin{array}{l}\text { - El equipo está conformado por } \\
\text { solo hombres, así que todas las } \\
\text { actividades que realizamos las } \\
\text { hicimos todos }\end{array}$ \\
\hline
\end{tabular}




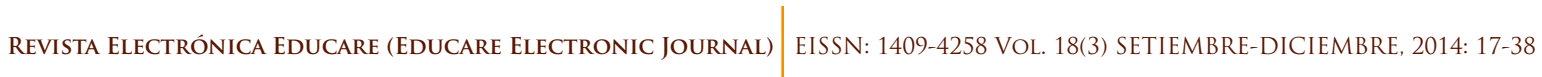

doi: http://dx.doi.org/10.15359/ree.18-3.2

URL: http://www.una.ac.cr/educare

CORREO: educare@una.cr

\begin{tabular}{|c|c|c|c|}
\hline $\begin{array}{l}\text { Nombre } \\
\text { del equipo }\end{array}$ & Hombres & Mujeres & Y el por qué \\
\hline Six pinos & $\begin{array}{l}\text { - Recolectar basura } \\
\text { - Regar árboles y } \\
\text { plantas }\end{array}$ & & $\begin{array}{l}\text { - En el equipo no hay mujeres, por } \\
\text { eso las actividades las hicimos los } \\
\text { hombres }\end{array}$ \\
\hline $\begin{array}{l}\text { Linternas } \\
\text { verdes }\end{array}$ & $\begin{array}{l}\text { - Plantar árboles } \\
\text { - Recoger basura y } \\
\text { colocarla en la bolsa } \\
\text { - Encalar árboles } \\
\text { - Regar y enderezar } \\
\text { árboles }\end{array}$ & & - En el equipo somos puros hombres \\
\hline Huellitas verdes & & $\begin{array}{l}\text { - Limpiar áreas verdes } \\
\text { - Plantar árboles y separar } \\
\text { basura, } \\
\text { - Hacer una composta }\end{array}$ & $\begin{array}{l}\text { - Realizamos estas actividades solo } \\
\text { las mujeres ya que en nuestro } \\
\text { equipo no hay ningún hombre }\end{array}$ \\
\hline
\end{tabular}

Nota: Elaboración propia.

Se observa en la tabla 4 que tanto hombres como mujeres realizan actividades ambientales; sin embargo, se encontró que en los equipos mixtos, en la mayor parte, los varones realizaron el trabajo más pesado y las féminas el menos cansado. Esto, según sus propias palabras, debido a que los masculinos tienen más fuerza y no les tienen miedo a los animales. Ellos dijeron que veían mal que las mujeres hicieran trabajo pesado y que había que protegerlas para no ocasionar accidentes. Las autoras Matamala y Rodríguez (2010) afirman que el distintivo de los hombres es la fuerza física, y los "describen como personas 'racionales', que 'entregan cariño y protección a las mujeres', que son 'duros', 'valientes', ... 'decididos' y que 'no deben mostrar debilidad' " (p. 74). Lamas (1986) menciona que "en muchas partes se suele valorar la fuerza sobre la debilidad, y se considera que los varones son los fuertes y las mujeres las débiles" (p. 195).

Testimonios de los equipos mixtos donde el hombre realiza el trabajo pesado:

Las mujeres levantamos basura, regamos las plantas, podamos los arbustos, los hombres levantaron basura, hicieron los hoyos y plantaron las flores. Porque lo decidimos así ya que estas actividades son para mujeres y las otras son para hombres y así todos colaboramos (Equipo Ecologistas del mundo).

Todos los integrantes participamos por igual, sin embargo, el hombre hizo los trabajos más pesados como cargar cubetas con agua y las bolsas de basura. Las mujeres recogen la basura y quitan la yerba de los arbolitos y participan más en las pláticas con los niños ya que es más concientizar a niños pequeños que son el futuro de la sociedad (Equipo Los rabanitos).

Sin embargo, en algunos equipos mixtos tanto hombres como mujeres realizan las mismas actividades, porque consideran que todos son iguales, porque avanzan más rápido y porque se dividen el trabajo de forma equitativa. En este caso los hombres no mostraron las características que mencionaron las autoras Matamala y Rodríguez (2010). Sin embargo, ellas 
doi: http://dx.doi.org/10.15359/ree.18-3.2

URL: http://www.una.ac.cr/educare

CORREO: educare@una.cr

también mencionan que hay una identidad de género menos arraigada donde los hombres y las mujeres comparten prácticas o roles más similares.

Los hombres y las mujeres hicimos lo mismo cortar pasto, podar árboles, recolectar basura, hacer cepas, echamos cal a los árboles, regamos plantas, plantamos flores. Todos hemos sido equitativos en todas las actividades para evitar problemas. Nosotros nos consideramos como iguales, todos hacemos de todo, no importa si somos mujeres u hombres (Equipo Lemontree).

\section{Motivos e interés para realizar acciones ambientales}

Los hombres y las mujeres tienen los mismos motivos, por los cuales realizan actividades ambientales; sin embargo, a un porcentaje más alto de féminas les gusta hacer las acciones ambientales para tener buena imagen y mantener limpio los espacios de la escuela; asimismo los hombres, pero en menor porcentaje. Tanto a féminas como varones les gusta que los espacios tengan buena presentación, y que las áreas no se vean maltratadas, descuidadas, sino estas deben ser agradables, bellas, limpias, amenas para que trasmitan bienestar y den buena impresión (ver tabla 5).

Tabla 5

Motivos por los cuales realizan actividades ambientales en la escuela, según sexo

\begin{tabular}{lcll}
\hline Hombres & $\%$ & Mujeres & $\%$ \\
\hline Por tener buena imagen & 23 & Por tener buena imagen & 27 \\
Por crear conciencia ambiental & 17 & Por cuidar y no desperdiciar el agua & 19 \\
Por cuidar y conservar el medio ambiente & 13 & Por cuidar y conservar el medio ambiente & 13 \\
Por asignación de actividades y obligación & 13 & Por crear conciencia ambiental & 11 \\
Por cuidar el agua & 8 & Por cumplimiento & 11 \\
Por difusión & 8 & Por asignación de actividades y obligación & 4 \\
Por preocupación sobre el medio ambiente & 6 & Por ser su escuela y hay que ayudarla & 4 \\
Otros & 12 & Otros & 11 \\
\hline
\end{tabular}

Nota: Elaboración propia.

Testimonios de estudiantes en donde mencionan por qué les gusta realizar actividades ambientales, principalmente cuando dicen por imagen y limpieza:

Para que tenga una mejor presentación, porque así el lugar tiene mejor imagen, para que la zona maltratada no se vea descuidada, porque considere necesario que esos lugares para mi deben ser agradables (bellos), para no ver áreas feas, para obtener una área verde, porque hay muchas áreas verdes maltratadas y hay que combatirlas (hombres del CBtis 212). 


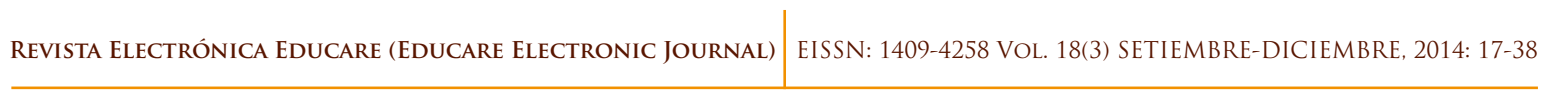

doi: http://dx.doi.org/10.15359/ree.18-3.2

URL: http://www.una.ac.cr/educare

CORREO: educare@una.cr

Porque me gusta que el espacio donde habito de un buen aspecto y esté limpio, lo hice ya que estaba demasiado sucio el lugar, para tener un ambiente más limpio, para que la visita y estancia en este lugar sea más agradable, además como es un lugar conocido también sirve para dar una buena impresión (mujeres del CBTis 212).

Testimonios de estudiantes que mencionan por qué les gusta realizar actividades ambientales principalmente cuando responden para cuidar el agua:

Porque era una forma de pedir que cuiden el agua, lo hice ya que en los sanitarios de las niñas se desperdicia mucho el agua. Para evitar el desperdicio del líquido vital y también evitar que la sigan contaminando, para que la gente ahorre el agua y no la desperdicie, el agua es muy esencial y es importante no desperdiciarla, para evitar el desperdicio de esta en algún lugar cercano, para tener más agua, porque sé que el agua cada vez está más contaminada y es necesario ahorrarla, porque yo relaciono mucho a Tetla con el cuidado del agua (mujeres del CBtis 212).

Para tener agua sin contaminante, lo hicimos para cuidar el agua, para que el agua se vea más limpia, porque el agua es muy valiosa para la vida (hombres del CBtis 212).

Al preguntar al estudiantado sobre qué acciones ambientales hacían con mayor entusiasmo, se encontró que hay diferencias entre hombres y mujeres ya que cada uno tiene sus propias preferencias y gustos. En la tabla 6 se pueden ver los problemas ambientales que más combatieron, con las acciones que más les agrada realizar. Las acciones que más les gusta hacer a los hombres son para mitigar la contaminación de residuos sólidos y áreas verdes maltratadas y las acciones que menos les entusiasman son las relacionadas con el agua. A las mujeres les gusta realizar acciones para combatir problemas de áreas verdes maltratadas y desperdicio y contaminación de agua.

Tabla 6

Problemas ambientales que combaten alumnos con las acciones que más les agrada hacer

\begin{tabular}{llll}
\hline \multicolumn{1}{c}{ Hombres } & \multicolumn{2}{c}{ Mujeres } \\
\hline Problema ambiental & $\%$ & Problema ambiental & $\%$ \\
Contaminación de residuos sólidos & 79 & Áreas verdes maltratadas & 72 \\
Áreas verdes maltratadas & 79 & Desperdicio y contaminación de agua & 64 \\
Deforestación & 64 & Deforestación & 55 \\
Falta de valores ambientales & 57 & Contaminación por residuos sólidos & 55 \\
Contaminación visual & 43 & Falta de valores ambientales & 45 \\
Desperdicio y contaminación de agua & 36 & Contaminación visual & 27 \\
\hline
\end{tabular}

Nota: Elaboración propia. 
doi: http://dx.doi.org/10.15359/ree.18-3.2

URL: http://www.una.ac.cr/educare

CORREO: educare@una.cr

En la tabla 7 se pueden ver, específicamente, las acciones ambientales que más les agrada realizar, según problema ambiental. Por ejemplo, tenemos que a ambos (hombre y mujer) les gusta recolectar la basura, plantar árboles, pegar carteles; pero a las mujeres, además, les gusta repartir volantes y folletos, pintar letreros y plantar plantas de ornato. A pesar del sexo, se identifican ambos realizando actividades semejantes, lo que demuestra que la mujer, a pesar de su condición física, es capaz de hacer las actividades que realizan y les gustan a los hombres.

Tabla 7

Acciones ambientales que más les gusta realizar a estudiantes del CBTis, según problema ambiental y sexo

\begin{tabular}{|c|c|c|c|}
\hline \multicolumn{2}{|l|}{ Hombres } & \multicolumn{2}{|l|}{ Mujeres } \\
\hline Problema ambiental & $\%$ & Problema ambiental & $\%$ \\
\hline Contaminación de residuos sólidos & 100 & Contaminación de residuos sólidos & 100 \\
\hline - Recolectar de basura & 82 & - Recoger basura & 67 \\
\hline - Limpiar la fuente & 18 & - Limpiar la zona de la fuente & 17 \\
\hline & & - Hacer campaña de pilas alcalinas & 16 \\
\hline Áreas verdes maltratadas & 100 & Áreas verdes maltratadas & 100 \\
\hline - Plantar árboles & 36 & - Plantar plantas de ornato y árboles & 50 \\
\hline - Colocar carteles & 18 & - Regar y podar arbustos & 25 \\
\hline - Mantener áreas verdes & 36 & - Arreglar cepas de los árboles & 13 \\
\hline - Pintar árboles y limpiar el área & 10 & - Quitar pasto y hierba & 12 \\
\hline Deforestación & 100 & Deforestación & 100 \\
\hline - Plantar arbolitos & 89 & - Plantar árboles y plantas de ornato & 67 \\
\hline - Pintar árboles & 11 & - Regar árboles y cuidarlos & 17 \\
\hline & & - Colocar carteles en las zonas verdes & 16 \\
\hline Desperdicio y contaminación de agua & 100 & Desperdicio y contaminación de agua & 100 \\
\hline - Poner carteles sobre el agua & 40 & - Pegar carteles en la escuela & 43 \\
\hline - Colaborar con las autoridades para evitar el desper- & 40 & - Reportar fugas de agua & 29 \\
\hline dicio del agua & & - No tirar agua de las llaves del baño & 14 \\
\hline $\begin{array}{l}\text { - Solicitar a las dependencias el apoyo para cuidar el } \\
\text { agua }\end{array}$ & 20 & - Llamar la atención a quienes la desperdician & 14 \\
\hline Contaminación visual & 100 & Contaminación visual & 100 \\
\hline - Recolectar la basura & 50 & - Pintar letreros & 67 \\
\hline - Limpiar la zona de la fuente & 17 & - Retirar la propaganda de postes & 33 \\
\hline - Quitar carteles & 17 & & \\
\hline - Arreglar los carteles & 16 & & \\
\hline Falta de valores ambientales & 100 & Falta de valores ambientales & 100 \\
\hline - Poner carteles & 38 & - Repartir volantes y folletos & 40 \\
\hline - Limpiar el salón y pasillos & 25 & - Hablar sobre los valores & 20 \\
\hline - Concientizar al alumnado & 25 & - Poner señalamientos de madera & 20 \\
\hline - Demostrar la importancia de tener un lugar limpio & 12 & - Llamar la atención & 20 \\
\hline
\end{tabular}

Nota: Elaboración propia. 
doi: http://dx.doi.org/10.15359/ree.18-3.2

URL: http://www.una.ac.cr/educare

CORREO: educare@una.cr

La causa principal por la cual les gusta realizar acciones ambientales tanto a hombres como a mujeres fue la diversión; la segunda, por la imagen de la escuela. El porcentaje de hombres es mayor que el de las mujeres, quizás porque las mujeres siempre demostraron ser más responsables en sus labores ambientales (ver tabla 8). Estas respuestas nos indican que la mejor manera de llevar la educación ambiental en las instituciones debe ser de manera lúdica, en la cual los estudiantes y las estudiantes se involucren con la naturaleza, disfrutando cada actividad que realicen para mitigar los problemas de deterioro ambiental.

Tabla 8

El porqué les gustó hacer mucho las acciones ambientales

\begin{tabular}{lcc}
\hline & Hombres & Mujeres \\
\hline & $\%$ & $\%$ \\
\hline Porque nos divertíamos & 35 & 24 \\
Para tener buena imagen & 25 & 14 \\
Para crear conciencia ambiental & 6 & 6 \\
Por la convivencia y participación de los niños & 5 & 10 \\
Por hacer algo por el ambiente & 0 & 14 \\
Por la planeación del programa & 3 & 8 \\
Por cuidar el agua & 5 & 6 \\
Por cuidar el medio ambiente & 3 & 6 \\
Por el contacto con la naturaleza & & 12 \\
Por ser fáciles de hacer & & 6 \\
otros & 18 & 6 \\
\hline
\end{tabular}

Nota: Elaboración propia.

Testimonios de estudiantes que mencionaron su gusto por realizar actividades ambientales, principalmente cuando mencionan por la diversión:

Porque fue divertido y fue algo que ayudo al medio ambiente, porque nos pusimos a jugar debajo de los aspersores de agua, porque no se nos dificulto, porque vimos muchas cosas y lo disfrutamos mucho, porque al estar encalando nos pasaron cosas chistosas, fue divertido mover la tierra y aprender a plantar arbolitos, porque en el transcurso del viaje nos pasaron muchas cosas y nos divertimos juntos, porque fue fácil y me divertí con mi equipo, fue muy entretenido, me divertí mucho con mis compañero, fue una actividad entretenida (Mujeres del CBTis 212). 
doi: http://dx.doi.org/10.15359/ree.18-3.2

URL: http://www.una.ac.cr/educare

CORREO: educare@una.cr

Al preguntarles si les gustaría vivir de nuevo la experiencia de realizar acciones ambientales en su escuela, para mitigar los problemas ambientales, un $45 \%$ de mujeres dijo mucho y los hombres solamente el $14 \%$. ¿Esto indica que a las mujeres les gusta cuidar más el medio ambiente o hacer acciones para el cuidado y conservación de los recursos naturales? A los hombres, a pesar de que participaron y cumplieron con las actividades asignadas, no les gustaría volverlo a repetirlo (ver tabla 9). La autora Murga (2009) menciona que las mujeres "se encuentran más sensibilizadas ante los problemas que afectan al medio físico... que indican el quebranto moral y ético de nuestras sociedades, aspectos... fundamentales en el modelo del desarrollo sostenible" (p. 180). Asimismo, Aguilar, Heredia y López $(2009$, p. 9) afirman "que las mujeres muestran una tendencia natural a las actitudes favorables con respecto al medio ambiente, mientras que en los hombres dichas actitudes se dan en un nivel más bajo". Figueroa $(2013$, p. 86) plantea que "las mujeres han sido, tradicionalmente, preservadoras, administradoras y suministradoras de los recursos naturales, muchas veces sin poder tener control sobre ellos". Bifani-Richard (2003) arguye que las mujeres son especialmente vulnerables a la escasez o degradación del medio ambiente, lo que hace que asuman una mayor responsabilidad para participar en programas de conservación y rehabilitación de los recursos naturales.

Tabla 9

Les gustaría vivir de nuevo esta experiencia

\begin{tabular}{cccc}
\hline Sexo & $\begin{array}{c}\text { Mucho } \\
\%\end{array}$ & $\begin{array}{c}\text { Poco } \\
\%\end{array}$ & $\begin{array}{c}\text { Nada } \\
\%\end{array}$ \\
\hline Hombres & 14 & 79 & 7 \\
Mujeres & 45 & 55 & 0 \\
\hline
\end{tabular}

Nota: Elaboración propia.

Con los argumentos anteriores podemos afirmar que a las mujeres del bachillerato les gusta cuidar y conservar más su medio ambiente, en comparación con los hombres; ya que ellas muestran mayor interés en hacer las acciones ambientales. Los testimonios del estudiantado nos dicen que les gusta cuidar su ambiente porque valoran y cuidan su naturaleza, contribuyen al medio ambiente, aprenden a crear conciencia ambiental y porque es divertido:

Porque es bonito que pueda yo contribuir a tener un ambiente limpio y en buen estado, además de que vive uno muchas experiencias. Porqué se siente lindo el hacer algo por la naturaleza, así aprendemos a crear conciencia ambiental, fue interesante y divertida, pero me hubiera gustado tener más tiempo y recursos. Porque se viven y se ven cosas que nunca me había tocado vivir con personas que hacen cosas divertidas (Mujeres del CBTis 212).

Porque hicimos algo por el ambiente, esto nos ayuda a poder valorar a nuestra naturaleza, a nuestro alrededor y cuidarla de todo tipo de contaminantes ya sean sólidos o líquidos (Hombres del CBTis 212). 


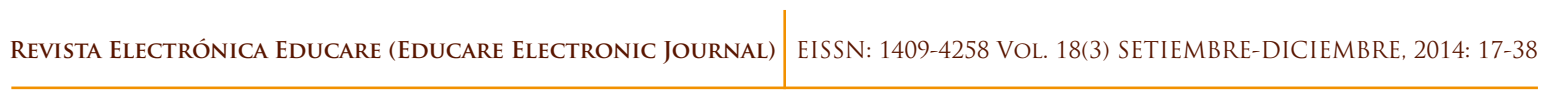

doi: http://dx.doi.org/10.15359/ree.18-3.2

URL: http://www.una.ac.cr/educare

CORREO: educare@una.cr

\section{Conclusiones}

Los programas ambientales son un instrumento para que el estudiantado innove y realice acciones ambientales de forma lúdica, para combatir los problemas, al respecto, de su escuela; asimismo para rescatar el papel de cambio que ellos tienen para la promoción de la educación ambiental, desde la perspectiva de género. Para realizar las acciones ambientales, la mayor parte de los equipos se conformaron por ambos sexos, lo que hace que haya una división del trabajo según sexo y preferencias en las actividades. Se aprovechan las capacidades, las habilidades y las preferencias que tienen los hombres y las mujeres para mitigar problemas ambientales como la contaminación de residuos sólidos y áreas verdes.

Las mujeres del nivel medio superior tienen más cualidades para realizar actividades para combatir el desperdicio y contaminación de agua y contaminación visual. De igual manera, se caracterizan por ser más disciplinadas, responsables, colaborativas, emotivas y tener mayor capacidad para controlar y liderar tareas ambientales.

Por naturaleza, la mayor parte de los equipos se conforman por hombres y mujeres para realizar acciones ambientales, cuando esto sucede los integrantes se dividen el trabajo de dos formas: a) los hombres realizan el trabajo más pesado y las mujeres el menos cansado, esto debido a la fuerza física del hombre, a la valentía que ellos tienen al enfrentar problemas de miedo y porque les gusta proteger a la mujer, b) tanto hombres como mujeres realizan las mismas actividades, porque se consideran que todos son iguales, avanzan más rápido y porque se dividen el trabajo de forma equitativa.

Cuando se conforman equipos solamente de hombres o mujeres, entonces, todos y todas realizan las mismas actividades, incluso las mujeres proponen plantar árboles en su PA, aunque sepan que necesitan mayor fuerza para hacerlo; sin embargo, ellas lo efectúan sin ninguna dificultad, ya que consideran que el hombre no es necesario para lograr sus objetivos.

A la mayor parte de las mujeres las motiva realizar acciones ambientales para tener buena imagen y mantener limpio los espacios de la escuela, cuidar y no desperdiciar el agua. A los varones les motiva más crear una conciencia ambiental.

El interés por el agua y lo estético del ambiente es un asunto que preocupa a hombres y mujeres como humanidad; sin embargo, es importante tomar en cuenta que en la cuestión ambiental, las problemáticas y sus efectos son distintas para hombres y mujeres, pues a ambos corresponden funciones diferenciadas en el manejo de los recursos naturales y también difiere su relación cotidiana con el entorno (la vida cotidiana).

Las mujeres del nivel medio superior se ven condicionadas por estereotipos de género y siguen reproduciendo roles tradicionales que se reflejan e impactan en los hombres al presentar menor interés en participar y en realizar acciones ambientales. Ante esto es importante lo que menciona Aguilar et al. (2009, p. 10): los resultados obtenidos son un factor "para propiciar la 
doi: http://dx.doi.org/10.15359/ree.18-3.2

URL: http://www.una.ac.cr/educare

CORREO: educare@una.cr

igualdad o, por lo menos, la mayor unificación de valores y comportamientos en los individuos que conforman una comunidad".

Aunque el autor Fortson (2003), propone que el sistema educativo deberá considerar que las nuevas condiciones en las tareas ambientales implican reconocer que "la problemáticas y sus efectos [en el medio], son distintos para hombres y mujeres, pues a ambos corresponden roles diferenciados en el manejo de los recursos naturales, como también difiere su relación cotidiana con el entorno" (p. 1).

En suma, el presente trabajo ofrece información para que el aprendizaje de la educación ambiental pueda llevarse a cabo con perspectiva de género. De esta manera, se podrían dejar atrás ciertas creencias y estereotipos de género que dualizan los intereses y las inquietudes de hombres y mujeres por el cuidado ambiental, además para alejar una visión esencialista donde las mujeres "por naturaleza" son propensas a proteger la naturaleza y los hombres funcionan como "protectores" de las mujeres.

\section{Referencias}

Aguilar, Á. M., Heredia, N. G. y López, G. E. (2009). Perspectiva de género en la educación formal como factor en la modificación de actitudes a favor de la conservación ambiental. En Memoria del X Congreso Nacional de Investigación Educativa (pp. 1-11). Veracruz, México. Recuperado de http://www.comie.org.mx/congreso/memoriaelectronica/v10/pdf/area_ tematica_03/ponencias/1711-F.pdf

Álvarez-Gayou, J. L. (2012). Cómo hacer investigación cualitativa: Fundamentos y metodología. México: Paidós.

Aranda, J. M. y García A. T. (2007). Perspectiva de género para el análisis de la participación femenina en organizaciones ambientalistas: El caso de la organización de mujeres ecologistas de la Sierra de Petatlán, Gro. Territorios, 16-17, 107-125. Recuperado de http:// dialnet.unirioja.es/servlet/articulo?codigo $=2924377$

Arellano, R. (2003). Género, medio ambiente y desarrollo sustentable: Un nuevo reto para los estudios de género. Revista de estudios de género, La Ventana, 17, 79-106. Recuperado de http://www.redalyc.org/pdf/884/88401705.pdf

Bifani-Richard, P. (2003). Algunas reflexiones sobre la relación género-medio ambiente. Revista de estudios de género. La Ventana, 17, 7-42. Recuperado de http://148.202.18.157/sitios/ publicacionesite/pperiod/laventan/Ventana17/17-1.pdf

Cáceres, G. y López, C. E. (setiembre-diciembre, 2009). El liderazgo femenino ambiental en Mérida, Venezuela. Fermentum Revista venezolana de Sociología y Antropología, 19(56), 570-595. Recuperado de http://www.redalyc.org/articulo.oa?id=70517761009 


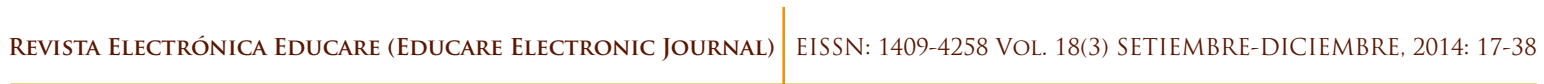

doi: http://dx.doi.org/10.15359/ree.18-3.2

URL: http://www.una.ac.cr/educare

CORREO: educare@una.cr

Colombara, M. (2006) Género, ambiente y desarrollo. Desde caminos paralelos hacia la transversalidad. Revista Geográfica Venezolana, 47(2), 157-186. Recuperado de http:// www.saber.ula.ve/bitstream/123456789/24685/2/articulo1.pdf

Espejel, A. (2009). Problemas ambientales, procedimiento metodológico y acciones de mitigación en el estado de Tlaxcala ( $2^{\mathrm{a}}$ ed.). Tlaxcala: Universidad Autónoma de Tlaxcala.

Espejel, A.y Flores, A. (octubre-diciembre, 2012). Educación ambiental escolar y comunitaria en el nivel medio superior. Puebla-Tlaxcala, México. Revista Mexicana de investigación educativa, 17(55), 1173-1199. Recuperado de http://www.redalyc.org/pdf/140/14024273008.pdf

Figueroa, M. E. (enero-junio, 2013). Construyendo lo que viene. Representaciones sociales del futuro en la red género y medio ambiente. Trayectorias, 15(36), 83-105. Recuperado de http://www.redalyc.org/articulo.oa?id=60727448005

Fortson, J. (2003). (Coord.). Equidad de género y medio ambiente. México: Inmujeres. Recuperado de http://www.cubaenergia.cu/genero/ambiente/a3.pdf

González, E. (2007). Educación ambiental: Trayectorias, rasgos y escenarios. México: Plaza y Valdés Editores.

Joekes, S., Green, C.y Leach, M. (2004). La integración del género en la investigación y las políticas ambientales. En V. Vázquez y M. Velásquez (Comps.), Miradas al futuro. Hacia la construcción de sociedades sustentables con equidad de género (pp. 487-564). México: UNAM.

Lamas, M. (1986). La antropología feminista y la categoría "género". Nueva antropología, 8(30), 173-198. Recuperado de http://www.redalyc.org/articulo.oa?id=15903009

Martínez, R. (enero-junio, 2010). La importancia de la educación ambiental ante la problemática actual. Revista Electrónica Educare, 14(1), 97-111.Recuperado de http://www.revistas.una. ac.cr/index.php/EDUCARE/article/view/1513

Matamala, M. L. y Rodríguez, M. C. (2010). Estudio exploratorio sobre la identidad de género de hombres adolescentes pertenecientes al sector barrio norte de concepción. Última Década, 33, 61-84. doi: http://dx.doi.org/10.4067/S0718-22362010000200005

Murga, M. (2009). Sobre las diferencias de género en la percepción social del desarrollo sostenible. Estudio empírico en estudiantes universitarios de alto rendimiento. Revista de Investigación Educativa, 27(1), 169-183. Recuperado de http://www.redalyc.org/articulo. oa?id=283322804010

Pérez, J. C. (2003). La variable de la masculinidad en los procesos para el desarrollo sustentable, experiencia y marco teórico. Revista de estudios de género. La ventana, 17, 250-302. Recuperado de http://www.redalyc.org/articulo.oa?id=88401710 
doi: http://dx.doi.org/10.15359/ree.18-3.2

URL: http://www.una.ac.cr/educare

CORREO: educare@una.cr

Soares, D. (enero-abril, 2007). Acceso, abasto y control del agua en una comunidad indígena chamula en Chiapas: Un análisis a través de la perspectiva de género, ambiente y desarrollo. Región y sociedad, 19(38), 25-50. Recuperado de http://www.scielo.org.mx/ scielo.php?script=sci_arttext\&pid=S1870-39252007000100002\&lng=es\&tIng=es

Soares, D., Castorena, L. y Ruiz, E. (julio-diciembre, 2005). Mujeres y hombres que aran en el mar y en el desierto: Reserva de la Biosfera El Vizcaíno, B.C.S. Frontera Norte, 17(34), 67-102. Recuperado de http://www.redalyc.org/articulo.oa?id=13603403

Taylor, S. J. y Bogdan, R. (1987). Introducción a los métodos cualitativos de investigación. La búsqueda de significados. Barcelona: Paidós.

Tuñón, E. (2000). La categoría género: Discusión, preguntas y nuevas propuestas. En Léxico de Política. México: UNAM/ Facultad Latinoamericana de Ciencias Sociales.

\section{Cómo citar este artículo en APA:}

Espejel-Rodríguez, A., Flores-Hernández, A. y Castillo-Ramos, I. (setiembre-diciembre, 2014). Educación ambiental en el nivel medio superior, desde la perspectiva de género, Tlaxcala, México. Revista Electrónica Educare, 18(3), 17-38. doi: http://dx.doi.org/10.15359/ree.18-3.2

Nota: Para citar este artículo en otros sistemas puede consultar el hipervínculo "Como citar el artículo" en la barra derecha de nuestro sitio web: http://www.revistas.una.ac.cr/index.php/EDUCARE/index 